\title{
Acknowledgement to Reviewers of Soil Systems in 2018
}

Soil Systems Editorial Office

MDPI, St. Alban-Anlage 66, 4052 Basel, Switzerland

Published: 11 January 2019

Rigorous peer-review is the corner-stone of high-quality academic publishing. The editorial team greatly appreciates the reviewers who contributed their knowledge and expertise to the journal's editorial process over the past 12 months. In 2018, a total of 69 papers were published in the journal, with a median time to first decision of 21 days and a median time to publication of 54 days. The editors would like to express their sincere gratitude to the following reviewers for their cooperation and dedication in 2018:

\begin{tabular}{ll} 
Ahmed, Ashour & Christiansen, Jesper Riis \\
Alava, Juan José & Churchman, Jock \\
Amonette, James E. & Courty, Pierre-Emmanuel \\
Antinoro, Chiara & Coyne, Mark \\
Arora, Bhavna & De Goede, Ron \\
Baker, Ian & Dewan, Ashraf M. \\
Bansal, Sheel & Dhiman, Saurabh \\
Bátková, Kamila & Doube, Bernard \\
Bene, Claudia Di & Duan, Luchun \\
Bento-Gonçalves, António & Duarte, Regina M.B.O. \\
Ben-Zvi, Rami & Dumon, Mathijs \\
Bhowmik, Arnab & Dwivedi, Dipankar \\
Blank, Robert & Eivazi, Frieda \\
Błońska, Ewa & Elzinga, Evert \\
Bölscher, Tobias & Fan, Xingwang \\
Brinton, William & Felde, Vincent \\
Bufo, Sabino Aurelio & Fernández, Manuel Pulido \\
Capek, Petr & Fernandez-Ahumada, Elvira \\
Carranca, Corina & Ferré, Ty \\
Cavalazzi, Barbara & Finley, Sheree J. \\
Cavani, Luciano & Fodor, Ferenc \\
Cerdà, Artemi & Foereid, Bente \\
Chao, Zhou & Frossard, Emmanuel \\
Chen, Chunmei & Fuchslueger, Lucia \\
Chen, Jui-Sheng & Furukawa, Yuichiro \\
Chendev, Yury & Garcia, Rosario \\
Cherubin, Maurício Roberto & Gasparatos, Dionisios \\
\hline & \\
\hline
\end{tabular}


Gelsomino, Antonio

Gemtos, Theofanis

Gent, Martin

Ghani Zadeh, Hossein

Gianni, Pes

Golabi, Mohammad H.

Gonneau, Cédric

Griepentrog, Marco

Gross, Avner

Hackenberger, Davorka K

Han, Zhong

Hassan, Sayed

$\mathrm{He}$, Bin

Henning, Jeremiah A

Hoffmann, Markus M.

$\mathrm{Hu}$, Pengjie

Huang, Wenjuan

Hurisso, Tunsisa T.

Hursthouse, Andrew

Jacomini, Carlo

Jandl, Robert

Jiménez-Ballesta, Raimundo

Johnson, Jane

Jones, Adele M

Józefowska, Agnieszka

Juck, David

Jung, Haesung

Kang, Namgoo

Karimian, Niloofar

Karlen, Douglas L.

Kavvadias, Victor

Kim, Choonsig

Kim, Christopher

Klumpp, Katja

Knelman, Joseph E.

Kopeć, Michał

Ladeira, Francisco Sergio Bernardes

Lafferty, Brandon

Lang, Rong

Lawrence, Corey

Le Bissonnais, Yves

Leinweber, Peter

Leiva-Brondo, Miguel
Li, Zijian

Lipson, David

Liu, Lei

Mahbub, Khandaker Rayhan

Manfreda, Salvatore

Mariussen, Espen

Martini, Edoardo

Matosziuk, Lauren

McDaniel, Marshall

Moreno, Eduardo

Mueller, Carsten

Nauer, Philipp

$\mathrm{Ng}$, Justin P.

Nguyen, Van Khanh

Núñez, Remigio Paradelo

Orzolek, Michael D.

Palke, Aaron

Palma, Luca Di

Pfahler, Verena

Pfeiffer, Birgit

Picoloto, Rochele S.

Pierluigi, Brandolini

Ponge, Jean-François

Pourret, Olivier

Rada, Elena Cristina

Raff, Jonathan

Ramos Miras, Jose Joaquin

Raub, Florian

Ray, Richard P.

Redeker, Kelly

Rietra, Rene

Rodrigo Comino, Jesús

Rodríguez-Seijo, Andrés

Rosaria, Sciarrillo

Rosenfeld, Carla E.

Różyło, Krzysztof

Salvati, Luca

Sanchez, Miguel

Sardini, Paul

Sarker, Tushar C.

Scarciglia, Fabio

Schaefer, Michael V

Scheckel, Kirk 
Schindlbacher, Andreas

Schlüter, Steffen

Schneider, Anna

Schütze, Claudia

Segvic, Branimir

Semenov, Vyacheslav

Sengupta, Aditi

Serio, Carmine

Sharma, Rajesh

Shuster, Jeremiah

Siebecker, Matthew G.

Sihi, Debjani

Siitari-Kauppi, Marja

Sipos, Péter

Skwarzec, Bogdan

Smagin, Andrey Valentinovich

Smith, Carol

Soman, Chinmay

Speratti, Alicia B.

Stavi, Ilan

Stromberger, Mary

Sun, Wu

Taghizadeh-Mehrjardi, Ruhollah

Tatarinov, Fedor

Teh, Yit Arn

Thomas, Sean

Thompson, Karen

Tian, Bin

Tositti, Laura
Trögl, Josef

Uteau, Daniel

Vallati, Andrea

van Hullebusch, Eric

Viccione, Giacomo

Vicente-Vicente, José Luis

Vidal, Alix

Vingiani, Simona

Virzo De Santo, Amalia

Vogel, Cordula

Von Haden, Adam

Von Sperber, Christian

Wang, Daoyuan

Wang, Fuyao

Ward, Ellen

Weyers, Sharon

Wiesmeier, Martin

Wilson, Susan

Wutzler, Thomas

Xenakis, Georgios

Xiao, Boqi

Young, Eric

Young, Robert B.

Zhamsueva, Galina S.

Zhang, Chao

Zhang, Guang-Hui

Zhang, Yulan

Żyromski, Andrzej

(C) 2019 by the authors. Licensee MDPI, Basel, Switzerland. This article is an open access article distributed under the terms and conditions of the Creative Commons Attribution (CC BY) license (http://creativecommons.org/licenses/by/4.0/). 